\title{
Aprendizaje autodirigido utilizando la estrategia didáctica Aprendizaje Basado en Proyectos
}

\author{
Development of Self-management through Project-Oriented \\ Learning strategy
}

\author{
Abril Paulina López Colón, Silvia Lizeth Olivares Olivares \\ y Miriam Lizzeth Turrubiartes Corolla
}

\section{Introducción}

La educación se dedica a facilitar en los alumnos la construcción de conocimientos y habilidades que les permitan desarrollar un proceso integral logrando una evolución continua dentro de los estándares curriculares establecidos por la SEP. Las reformas curriculares educativas representan un esfuerzo sostenido y orientado hacia una propuesta de formación integral de los alumnos, cuya finalidad es el desarrollo de competencias para la vida(SEP, 2011c).

Una parte esencial de la educación consiste en brindar a los alumnos experiencias que les permita desarrollar competencias. Esta idea surge de la necesidad de convertir a los estudiantes en personas autónomas capaces de enfrentar situaciones problemáticas que les plantea la vida y el entorno (Salgado, Corrales, Muñoz, \& Delgado , 2012). El objetivo es contribuir a la enseñanza del aprender a aprender donde los niños estén preparados para un ambiente con posibilidades ilimitadas (Olivares Olivares, 2015).

El concepto de competencia en la educación se refiere a la comprensión y capacidad aprendidas para realizar tareas en diversos escenarios. Palmer, Montaño y Palou (2009) la definen como las acciones que el alumno tendrá que ser capaz de efectuar en un largo periodo de tiempo, integradas por conocimientos, habilidades y actitudes. Los autores contemplan los conocimientos teóricos y prácticos, así como las actitudes que van del saber y saber hacer al saber ser 0 estar.

El desarrollo de competencias genéricas contribuye de manera significativa a que los alumnos sean capaces de resolver situaciones problemáticas que les plantea la vida en su entorno. El término es referido a las habilidades necesarias para la vida teniendo como objetivo satisfacer plenamente las exigencias sociales. Villa y Poblete (2007) dividen las competencias genéricas en instrumentales, interpersonales y sistémicas. Las instrumentales se refieren a todos los procedimientos mentales y cognitivos. Las interpersonales son referidas a la expresión de sentimientos y compromiso social con los demás. Las sistémicas son los sistemas en totalidad como agrupación de un conocimiento.

La educación busca integrar los conocimientos, habilidades, destrezas, valores y actitudes para que el docente oriente 


\section{taphiya $\mathbf{4 6}$}

los aprendizajes de los alumnos. Con este enfoque el alumno tendrá mayores elementos para resolver problemas y adaptarse a las demandas que la sociedad exige. El profesor debe centrarse en detectar los estilos de aprendizaje de los alumnos, descubrir qué es lo que saben y lo qué les falta por saber de tal manera que pueda involucrarlos de manera activa en su propio aprendizaje (Tobón, 2006).

Los programas educativos están enfocados en el desarrollo de competencias genéricas centradas en el estudiante y su capacidad propia para aprender; el desarrollo del aprendizaje autodirigido implica utilizar las destrezas, conocimientos y actitudes necesarias para enfrentarse a situaciones en la vida cotidiana (Olivares \& López, 2015).

La capacidad de aprendizaje autodirigido es definida como "la participación activa del estudiante en el diseño, la conducción y la evaluación de un esfuerzo del aprendizaje, el cuál es elegido y llevado a cabo por el propio aprendiz" (Parra, Cerda, López-Vargas, \& Saiz, 2014, pág. 94). Los estudiantes son capaces de autodirigir o autorregular su aprendizaje, determinar las actividades que quiere realizar y decidir las metas que quiere cumplir.

Existen tres subcompetencias fundamentales para el proceso de autodirección en el alumno. En la tabla 1 se aprecia una comparación de las tres subcompetencias desarrolladas por tres diferentes autores, los cuales confirman que el proceso de autodirección es un procedimiento cíclico que abarca la participación total del aprendiz.

\begin{tabular}{|c|c|c|c|}
\hline Sub competencias & Olivaresy López (2015) & $\begin{array}{c}\text { Parra, Cerda, Lópezy } \\
\text { Saiz (2014) }\end{array}$ & $\begin{array}{c}\text { Varela, Durán y Fortual } \\
\text { (2013) }\end{array}$ \\
\hline $\begin{array}{l}\text { Planeación de } \\
\text { Actividades }\end{array}$ & $\begin{array}{l}\text { Planea sus actividades } \\
\text { detectando metas de } \\
\text { aprendizaje. }\end{array}$ & $\begin{array}{l}\text { Toma el control sobre el } \\
\text { contexto social a fin de } \\
\text { alcanzar el logro } \\
\text { académico deseado. }\end{array}$ & $\begin{array}{c}\text { Implica ser un } \\
\text { participante intencional } \\
\text { y activo, capaz de iniciar } \\
\text { y dirigir el propio } \\
\text { aprendizaje. }\end{array}$ \\
\hline Deseo de aprender & $\begin{array}{l}\text { Cuentan con actitudes } \\
\text { de entusiasmo y } \\
\text { responsabilidad por los } \\
\text { objetivos de } \\
\text { aprendizaje. }\end{array}$ & $\begin{array}{l}\text { Participa activamente de } \\
\text { forma permanente en } \\
\text { su aprendizaje. }\end{array}$ & $\begin{array}{l}\text { Mantiene una } \\
\text { motivación positiva y } \\
\text { constante. }\end{array}$ \\
\hline Reflexión crítica & $\begin{array}{l}\text { Identifica sus logros y } \\
\text { debilidades para } \\
\text { redirigir su aprendizaje }\end{array}$ & $\begin{array}{l}\text { Auto-observa sus } \\
\text { procesos cognitivos y } \\
\text { motivacionales durante } \\
\text { el proceso de } \\
\text { aprendizaje. }\end{array}$ & $\begin{array}{l}\text { Valora y realimenta de } \\
\text { manera oportuna los } \\
\text { aciertos y errores. }\end{array}$ \\
\hline
\end{tabular}

Tabla 2: Comparación de las sub competencias de cada modelo por autor.

Es posible afirmar que el aprendizaje autodirigido es un proceso estructural que comprende varias subcompetencias y depende de la participación del alumno. La primer subcompetencia es la planeación de actividades, en ella los alumnos fijan los objetivos que desean alcanzar al finalizar su proceso de aprendizaje. Los autores indicados en la Tabla 1 confirman que el aprendiz debe tomar el control total de su contexto para seleccionar y dirigir las metas de 


\section{tapbiya $\mathbf{4 6}$}

aprendizaje. La planeación, implica la selección de herramientas y recursos necesarios para el proceso de aprendizaje, además de dictaminar las actividades que va a llevar a cabo para cumplir con los objetivos planteados anteriormente.

El deseo de aprender se refiere al compromiso actitudinal que el estudiante posee durante el proyecto, implica una mente positiva a la disposición de actividades variadas y un alto grado de responsabilidad para cumplir con cada una de ellas (Cázares, 2009). El proceso de aprendizaje autodirigido depende totalmente del compromiso y actitud del que el alumno disponga; es decir, un alumno con una mente negativa y con poca disposición para ejecutar las actividades no logrará cumplir con las metas de aprendizaje del proyecto; en cambio, un estudiante con una buena actitud y disposición para realizar las actividades de aprendizaje logrará cumplir con los objetivos establecidos del proyecto (Vives-Varela, Durán-Cárdenas, Varela-Ruíz, \& Fortoul van der Goes, 2013).

La reflexión crítica es utilizada y categorizada por múltiples autores que la explican como un proceso de autorreflexión e implica que el aprendiz valore su propio proceso de aprendizaje, de tal manera que evalué cada una de las acciones y actividades que está llevando a cabo, esto con el fin de identificar sus logros y debilidades para redirigir su aprendizaje; la reflexión es un proceso de observación continua en la que el estudiante detecta sus errores y los modifica para alcanzar sus metas de aprendizaje (Tonnuci F. , 2001, pág. 39).

La estrategia didáctica utilizada durante la elaboración de este estudio para favorecer la autodirección del alumno, es el Aprendizaje Basado en Proyectos (ABPr). Los proyectos surgen de los intereses y dudas que el contexto provoca en los alumnos. El equipo de educación infantil y primer ciclo de primaria del CP Antzuola (2001, pág. 4) lo define como "una 0 varias actividades planteadas alrededor de un tema, cuyo objetivo es la construcción social de significados". Los proyectos fomentan y permiten el desarrollo de una conciencia crítica y la exploración de valores significativos.

El método basado en proyectos se deriva, según Arias Molina (2015), de un concepto de educación que rompe el esquema de educación tradicionalista en la que el estudiante es el centro del proceso de enseñanza-aprendizaje y el alumno aprende a vincular sus experiencias de vida con nueva información creando conceptos de aprendizaje significativos.

Según Olivares Olivares (2015), existen tres dimensiones para la implementación del aprendizaje autodirigido en el aula; Estrategias de Aprendizaje, Manejo de Emociones, y Mejora Continua. Las Estrategias de Aprendizaje se refieren al establecimiento de metas y a la planificación de las actividades que se llevarán a cabo para lograr los objetivos de trabajo. El manejo de emociones es referido a las estrategias de autocontrol y administración de tiempo, esfuerzo y búsqueda de conocimiento utilizando como principal factor la voluntad individual para aprender a construir lo que les interesa (Cázares, 2009). La mejora continua se refiere al desarrollo de la autonomía de los estudiantes, este concepto abarca la importancia de aprender de una manera profunda y de ser capaz de aplicar lo aprendido (Vives-Varela et al., 2013).

Con el objetivo de fomentar el aprendizaje autodirigido en los alumnos se buscaron estrategias pedagógicas adecuadas para su implementación en el salón de clases; la estrategia didáctica elegida es el Aprendizaje Basado en Proyectos (ABPr). Clardy (2000), argumenta que cuando se utilizan los proyectos reales de aprendizaje como base para el estudio, los factores contextuales y organizativos ejercen una influencia en la aparición de comportamientos de aprendizaje autodirigidos en los estudiantes.

En las instituciones educativas se puede describir la imperante necesidad de generar cambios en el aula para el desarrollo de competencias en los alumnos, pero ¿De qué forma la estrategia didáctica Aprendizaje Basado en Proyectos permite desarrollar la autodirección en alumnos de segundo grado de educación primaria?

Esta investigación busca dar respuesta a los presentes objetivos.

General. Determinar la forma en que la estrategia didáctica Aprendizaje Basado en Proyectos permite 


\section{tgphiyg $\mathbf{4 6}$}

desarrollar el aprendizaje autodirigido en alumnos de segundo grado de educación primaria. Específicos.

1. Medir el cambio de aprendizaje autodirigido en las dimensiones de Estrategias de Aprendizaje, Manejo de Emociones y Mejora Continua, a partir del método de Aprendizaje Basado en Proyectos.

2. Medir el proceso de aprendizaje autodirigido de los estudiantes a partir de las tres variables; Planeación de Actividades, Deseo de Aprender y Reflexión Crítica, durante la aplicación de la estrategia de Aprendizaje Basado en Proyectos.

La elaboración de este documento justifica la evidente necesidad de implementar prácticas docentes basadas en la autodirección del aprendizaje a través de la estrategia pedagógica de Aprendizaje Basado en Proyectos, el cual se introduce para ayudar a los estudiantes a entender como conectar sus intereses a los académicos a través de situaciones didácticas dentro del aula (Martínez \& McGrath, 2013).

\section{Método}

La población elegida para la elaboración de esta investigación se enfoca en alumnos de segundo año de educación primaria inscritos en un colegio particular ubicado en la localidad de San Luis Potosí. El grupo elegido está constituido por 18 alumnos, de los cuales 8 son niños y 10 son niñas. Los alumnos se encuentran en un rango de edad entre 6 a 8 años.

Los instrumentos utilizados durante esta investigación permitieron la colección de datos en el trabajo de campo para la obtención de resultados (Valenzuela \& Flores, 2011). Para la elaboración de este estudio se utilizaron dos instrumentos de medición:

1. Sección de Autodirección del Cuestionario de Competencias Genéricas Individuales de Olivares y López (2015), como un cuestionario de pre-test y post-test en los alumnos (instrumento cuantitativo).

2. Observación en el aula, con modalidad de participación moderada (instrumento cualitativo)

El primer instrumento utilizado es el Cuestionario de Competencias Genéricas Individuales de Olivares y López (2015); este instrumento es una herramienta eficaz para la detección de competencias en los individuos; es importante mencionar que el modelo conceptual del cuestionario cuenta con el valor de Alpha de Cronbach (0.659), asegurando su validez y credibilidad en el estudio.

Para su implementación fue necesario seleccionar la sección de aprendizaje autodirigido, esta permite detectar el cómo los alumnos perciben el aprendizaje y lo desarrollan; fue necesario adaptar las cuestiones para que los alumnos de primaria tuvieran un entendimiento comprensible y claro. El instrumento cuantitativo se baso en tres dimensiones, Estrategias de Aprendizaje, Manejo de Emociones y Mejora Continua. Dado que es una herramienta cuantitativa se interpreta numéricamente con la escala de Linkert del 1 a 5, indicando el 5 como mayor puntaje, y el 1 con menor puntaje.

El segundo instrumento de medición utilizado es la Observación en el aula. La aplicación de este instrumento fue utilizada como observación cualitativa, la cual pretende captar los sucesos de la realidad en forma natural con el propósito de describir de forma clara los acontecimientos sucedidos durante el desarrollo de las actividades propuestas (Hernández, Fernández, \& Baptista, 2010).

El instrumento se llevó a cabo de forma individual y por equipos. Para aplicar este instrumento fue necesario diseñar una rúbrica de acuerdo a las variables Planeación de Actividades, el Deseo de Aprender y la Reflexión Crítica. Las 
variables fueron evaluadas determinando un nivel de desempeño (alto, medio 0 bajo).

El trabajo de campo de esta investigación se realizó bajo el enfoque de poblaciones dependientes; el cual explica que la investigación se realiza con los mismos sujetos bajo condiciones diferentes con un pre-test y un post-test; el pre-test mide las habilidades que tienen los alumnos antes de la aplicación de las situaciones de aprendizaje planteadas por un investigador. El post-test, demuestra el cambio existente en las mismas habilidades entre los mimos sujetos después de la aplicación de las situaciones de aprendizaje (Valenzuela \& Flores, 2011).

La implementación de la estrategia ABPr fue aplicada del 28 de agosto al 8 de septiembre de 2017. Para una mayor organización en el trabajo de esta investigación se realizó un orden cronológico de trabajo el cual se describe en la Tabla 2.

\begin{tabular}{|c|c|c|}
\hline Fecha & Actividad & Desarrollo \\
\hline 28 de agosto de 2017 & $\begin{array}{l}\text { Aplicación del } \\
\text { pre-test }\end{array}$ & $\begin{array}{l}\text { Aplicación del instrumento cuantitativo sección de } \\
\text { Autodirección del Cuestionario de Competencias Genéricas } \\
\text { Individuales de Olivares y López (2015). }\end{array}$ \\
\hline $\begin{array}{l}28 \text { de agosto al } 8 \text { de } \\
\text { septiembre de } 2017\end{array}$ & $\begin{array}{l}\text { Implementación } \\
\text { de la estrategia }\end{array}$ & $\begin{array}{l}\text { Implementación de actividades con base a la estrategia } \\
\text { didáctica ABPr. }\end{array}$ \\
\hline $\begin{array}{l}28 \text { de agosto al } 8 \text { de } \\
\text { septiembre de } 2017\end{array}$ & $\begin{array}{l}\text { Evaluación } \\
\text { cualitativa }\end{array}$ & $\begin{array}{l}\text { Aplicación del instrumento cualitativo “Observación en el } \\
\text { aula”, se llevará a cabo durante las actividades que los } \\
\text { estudiantes realicen. }\end{array}$ \\
\hline $\begin{array}{l}8 \text { de septiembre de } \\
2017\end{array}$ & $\begin{array}{l}\text { Aplicación del } \\
\text { post-test }\end{array}$ & $\begin{array}{l}\text { Aplicación del instrumento cuantitativo, sección de } \\
\text { Autodirección del Cuestionario de Competencias Genéricas } \\
\text { Individuales de Olivares y López (2015). }\end{array}$ \\
\hline
\end{tabular}

Tabla 2: Orden cronológico del desarrollo del estudio.

El proyecto elegido consiste en que el alumno exponga la información sobre un tema de interés propio a través de la búsqueda de información en diversas fuentes; el objetivo es desarrollar la capacidad de reflexión creando metas para la construcción de una investigación realizando varias actividades planteadas alrededor de un tema.

Para la producción del proyecto fue necesario desarrollar una serie de documentos utilizados para el desarrollo de la estrategia ABPr: instrucciones para el estudiante, guía del profesor y la planeación del proyecto.

Las actividades autodirigidas propuestas bajo la estrategia Aprendizaje Basado en Proyectos, se resumieron en cuatro fases:

1. Presentación de la situación problema: Definición de conceptos fundamentales, elección de un tema de su interés, establecimiento de metas a lograr y elección de los equipos para trabajar.

2. Identificación de necesidades de aprendizaje: Establecimiento de las preguntas guía para la investigación de su tema y elección de recursos que utilizaron durante su investigación.

3. Aprendizaje de la información: Lectura de recursos, búsqueda y recolección de información, elaboración de borrador con información seleccionada e intercambio de ideas planteando un análisis reflexivo. 


\section{tgphiyg $\mathbf{4 6}$}

4. Resolución del problema e identificación de nuevos problemas: Elaboración de documento con información recuperada y apoyos gráficos para la presentación de su proyecto, sesión de intercambio de ideas acerca de los errores detectados y su posible solución.

Para una mejor descripción de la intervención educativa y la aplicación del instrumento cualitativo se desarrollo la tabla 3.

\begin{tabular}{|c|c|c|c|}
\hline $\begin{array}{c}\text { Fases de } \\
\text { implementación de la } \\
\text { estrategia ABPr. }\end{array}$ & $\begin{array}{l}\text { Actividades auto } \\
\text { dirigidas }\end{array}$ & $\begin{array}{l}\text { Integración de la autodirección en cada } \\
\text { actividad }\end{array}$ & $\begin{array}{c}\text { Sub-competencias } \\
\text { a evaluar }\end{array}$ \\
\hline $\begin{array}{l}\text { Presentación de la } \\
\text { situación problema } \\
\text { (28-29 de agosto) }\end{array}$ & $\begin{array}{l}\text { Metas esperadas } \\
\text { por los alumnos }\end{array}$ & $\begin{array}{c}\text { Actividades previas ¿Qué tengo que } \\
\text { planear o establecer? }\end{array}$ & \multirow{3}{*}{$\begin{array}{l}\text { Estrategias de } \\
\text { aprendizaje / } \\
\text { Planeación de } \\
\text { Actividades }\end{array}$} \\
\hline $\begin{array}{l}\text { Identificación de } \\
\text { necesidades de } \\
\text { aprendizaje } \\
\text { (30 de agosto-1 de } \\
\text { septiembre) }\end{array}$ & Preguntas guía & $\begin{array}{l}\text { Actividad de inicio ¿Con qué } \\
\text { información cuento? }\end{array}$ & \\
\hline \multirow{2}{*}{$\begin{array}{l}\text { Aprendizaje de la } \\
\text { información } \\
\text { (4-7 de septiembre) }\end{array}$} & $\begin{array}{l}\text { Actividades de } \\
\text { aprendizaje: } \\
\text { Lectura y } \\
\text { recolección de } \\
\text { información }\end{array}$ & $\begin{array}{c}\text { Actividad para identificar o reconocer } \\
\text { ¿Cuál es la mejor opción? }\end{array}$ & \\
\hline & $\begin{array}{l}\text { Apoyo } \\
\text { instruccional y } \\
\text { Ambiente de } \\
\text { aprendizaje }\end{array}$ & $\begin{array}{l}\text { Actividad para reflexión ¿Cómo me } \\
\text { siento al investigar acerca de un tema? } \\
\text { ¿Cuáles son los obstáculos que me } \\
\text { impiden resolver las preguntas } \\
\text { establecidas por mí? }\end{array}$ & $\begin{array}{l}\text { Manejo de } \\
\text { emociones / } \\
\text { Deseo de } \\
\text { Aprender }\end{array}$ \\
\hline $\begin{array}{c}\text { Resolución del } \\
\text { problema e } \\
\text { Identificación de nuevos } \\
\text { problemas } \\
\text { (7-8 de septiembre) }\end{array}$ & $\begin{array}{l}\text { Identificación de } \\
\text { recursos }\end{array}$ & $\begin{array}{l}\text { Actividad de cierre ¿qué recursos } \\
\text { tengo? ¿qué podría hacer mejor? } \\
\text { ¿trabaje en equipo? ¿ayude a resolver } \\
\text { las preguntas de mis compañeros? }\end{array}$ & $\begin{array}{l}\text { Mejora continua / } \\
\text { Reflexión Crítica }\end{array}$ \\
\hline
\end{tabular}

Tabla 3: Proceso de implementación de la estrategia didáctica.

Los datos fueron recolectados mediante técnicas de estadística t de student para evaluar el instrumento cuantitativo, y las notas de observación para evaluar el instrumento cualitativo. La estadística t de student permitió analizar los resultados cuantitativos de los estudiantes mediante la aplicación de un pre-test y post-test, permitiendo una comparación diferencial entre las medias de los alumnos del grupo. 
Los instrumentos cuantitativos y cualitativos utilizados en este estudio fueron determinados con el objetivo de obtener una triangulación de resultados; la triangulación, según Ikuda Benavides y Gómez-Restrepo (2005), se refiere al uso de métodos cuantitativos y cualitativos en el estudio de un fenómeno. La triangulación entre los instrumentos cuantitativos y cualitativos es una herramienta eficaz y enriquecedora que le confiere al documento una profundidad y complejidad adecuada para la consistencia de los hallazgos desarrollados en el presente estudio.

\section{Resultados}

\section{Resultados del instrumento cuantitativo}

Los resultados del cuestionario de aprendizaje autodirigido (instrumento cuantitativo) se dividieron entre un pre-test establecido antes de la aplicación del proyecto de aprendizaje y el post-test aplicado después del proyecto.

Fue necesario realizar el pre-test y post-test del Cuestionario de Competencias Genéricas Individuales, y comparar las medias comprobando si son igual o bien, no existe diferencia significativa. El cuestionario cuenta con 10 ítems considerando la escala Likert del 1 al 5 donde 5 era considerado como totalmente de acuerdo y 1 totalmente en desacuerdo; dentro del cuestionario se incluye un ítem con sentido negativo, en este caso la escala Likert se consideró a la inversa donde el valor favorable es 5 totalmente en desacuerdo.

\begin{tabular}{|c|c|c|c|c|c|c|}
\hline \multirow{2}{*}{ Dimensión } & \multirow{2}{*}{ Item } & \multicolumn{5}{|c|}{ Escala Likert } \\
\hline & & $T D$ & D & N & $A$ & $T A$ \\
\hline \multirow{2}{*}{$\begin{array}{c}\text { Estrategia de } \\
\text { aprendizaje }\end{array}$} & Tengo claras mis metas de aprendizaje en cada curso & 1 & 2 & 3 & 4 & 5 \\
\hline & Leer es algo que evito si es posible. & 5 & 4 & 3 & 2 & 1 \\
\hline \multirow{6}{*}{$\begin{array}{l}\text { Manejo de } \\
\text { emociones }\end{array}$} & Me gusta probar nuevas tecnologías. & 1 & 2 & 3 & 4 & 5 \\
\hline & Me siento feliz por lo que soy. & 1 & 2 & 3 & 4 & 5 \\
\hline & Sé reconocer los logros de los demás. & 1 & 2 & 3 & 4 & 5 \\
\hline & Intento superar mis debilidades. & 1 & 2 & 3 & 4 & 5 \\
\hline & Quiero aprender cosas nuevas. & 1 & 2 & 3 & 4 & 5 \\
\hline & Me gusta entender en qué me equivoqué en un examen & 1 & 2 & 3 & 4 & 5 \\
\hline \multirow{2}{*}{ Mejora continua } & Soy exitoso en mis estudios profesionales & 1 & 2 & 3 & 4 & 5 \\
\hline & Ajusto mis estrategias si no estoy alcanzando mis metas. & 1 & 2 & 3 & 4 & 5 \\
\hline
\end{tabular}

Donde TA significa Total Acuerdo, A: Acuerdo, N: Neutro, DA: Desacuerdo y TD: Total Desacuerdo.

Tabla 4: Codificación por nivel para cada ítem del cuestionario. 


\section{taphiya $\mathbf{4 6}$}

La estadística del cuestionario se elaboró con un nivel de confianza del 95\% donde el valor críitico de acuerdo al tamaño de muestra en este estudio es 1.739, con un grado de libertad de 17; el valor de prueba obtenido es de 2.123 por lo que se concluye que el valor de prueba es mayor que el valor crítico aumentando los niveles de autodirección entre ambos tests; se puede argumentar que, según los datos obtenidos, la estrategia de Aprendizaje Basada en Proyectos sí desarrolla el aprendizaje autodirigido.

Los resultados generales obtenidos en la prueba t student de ambos cuestionarios (pre-test y post-test) identificaron un p valor general de 0.04, lo cual indica que hay una diferencia significativa en el proceso de aprendizaje autodirigido de los estudiantes favoreciendo la competencia general planteada en el presente estudio.

La sección de autodirección del Cuestionario de Competencias Genéricas Individuales de Olivares y López (2015) se divide en tres dimensiones. Para la evaluación específica de cada dimensión del instrumento, se llevó a cabo una comparación con los resultados obtenidos de la prueba t student, detectando el p-valor y la interpretación obtenida de cada dimensión.

\begin{tabular}{ccc}
\hline Dimensión & p-valor & Interpretación \\
\hline Estrategias de aprendizaje & 0.020 & Sí hay diferencia significativa \\
\hline Manejo de emociones & 0.270 & No hay diferencia significativa \\
\hline Mejora continua & 0.003 & Sí hay diferencia significativa \\
\hline
\end{tabular}

Tabla 5: Resultados del P-valor obtenido de la prueba t student de las dimensiones aplicadas en el instrumento cuantitativo.

En la Tabla 5 se puede identificar que la dimensión de Mejora Continua es la que tiene mayor diferencia significativa entre las dimensiones evaluadas, seguida de la dimensión de Estrategias de Aprendizaje donde también existe diferencia significativa. Ambas dimensiones se caracterizaron por la definición de metas planteadas y el desarrollo de actividades para lograrlas. Al elegir un tema de interés, los estudiantes se sintieron motivados buscando información continua en distintas fuentes, inclusive fuera del contexto escolar.

La dimensión de Manejo de emociones, sin embargo, no logró adquirir una diferencia significativa durante la aplicación de la estrategia ABPr. Esto se debe a que algunos estudiantes no reconocían sus propias capacidades o se sentían intimidados al salir de su zona de confort sintiéndose dudosos al llevar a cabo las actividades de aprendizaje.

Se elaboró, además, una media estadística de cada una de las tres dimensiones para analizar los resultados y verificar la diferencia entre cada una de ellas. En la figura 3 se pueden apreciar los resultados adquiridos entre ambos test y las diferencias existentes entre las medias de los alumnos en el antes y el después del proyecto y las actividades aplicadas. 


\section{taphiya $\mathbf{4 6}$}

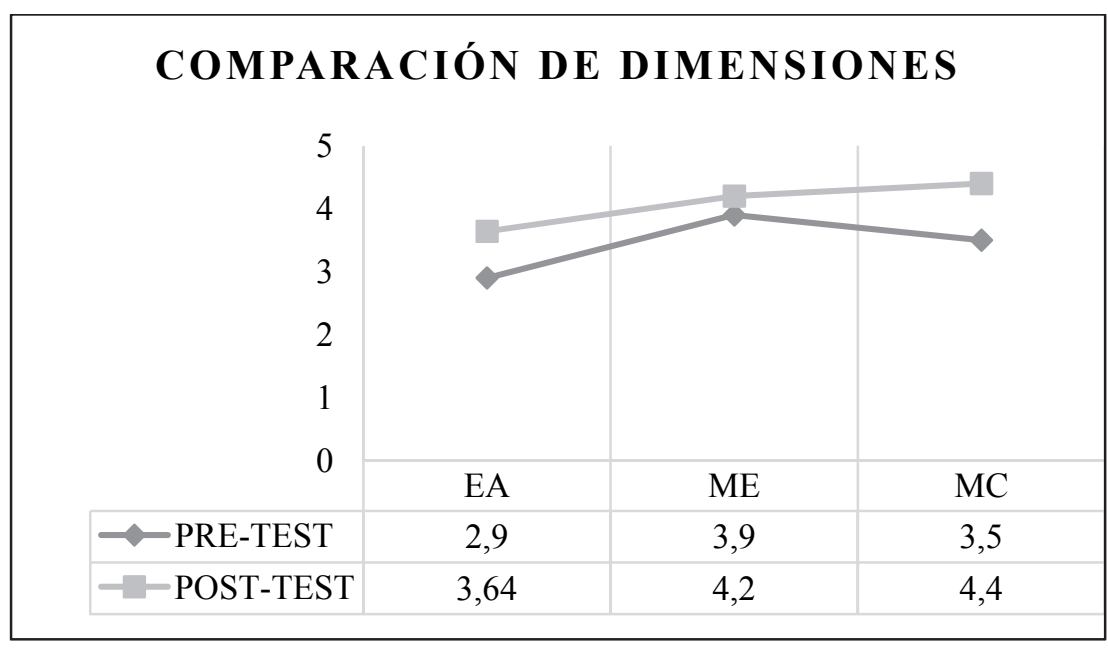

Figura 2: Gráfica de comparación de medias de las dimensiones aplicadas en el instrumento cuantitativo.

Con base a los resultados estadísticos obtenidos en la comparación de medias entre las tres dimensiones evaluadas descritas en la figura 2, se percibe la dimensión de Mejora Continua presenta que hay diferencia significativa con una media de 3.5 en el pre-test aumentando favorablemente a una media de 4.4 considerándola como la dimensión con mayor impacto en el desarrollo de la competencia de autodirección.

En la dimensión de Estrategias de Aprendizaje se logra apreciar que existe una diferencia significativa entre ambas medias aumentando de 2.9 a 3.6. Sin embargo, la dimensión de Manejo de Emociones demostró que no hay diferencia significativa entre ambas medias, teniendo un resultado de 3.9 a 4.2 notando una diferencia de 0.3 entre la aplicación de ambos tests.

Se puede argumentar entonces, que la dimensión con una mayor diferencia significativa fue la Mejora Continúa (MC)aumentando un 0.9 entre las medias de ambos tests a diferencia de las demás dimensiones.

Existió un cambio en los estudiantes posterior a las actividades planteadas; específicamente en la dimensión MC, la cual estipula que los alumnos tienen un mayor interés por aprender cosas nuevas para ellos y se sienten preparados para nuevos retos en actividades intelectuales. El grupo demostró capacidad de búsqueda de aprendizaje, logrando la autodirección de la misma; de igual manera, su rendimiento académico mejoró, no sólo en la materia plateada, sino en otras materias estipuladas en los planes de estudio de la SEP.

\section{Resultados del instrumento cuantitativo}

La aplicación del instrumento cualitativo se realizó con base a una rúbrica de acuerdo a las habilidades de autodirección; se dividió en variables que permiten visualizar la medición del cambio de autodirección en la Planificación de Actividades, Deseo de Aprender y Reflexión Crítica, a partir de la estrategia ABPr. Las variables fueron descritas y argumentadas en capítulos anteriores.

En la Tabla 6, se logran apreciar de manera específica las tres habilidades utilizadas en el proceso de observación y la forma en la que se determino el nivel de desempeño (alto, medio o bajo). 


\section{tapbiya $\mathbf{4 6}$}

\begin{tabular}{|c|c|c|c|}
\hline \multirow{2}{*}{ Habilidades de autodirección } & \multicolumn{3}{|c|}{ Nivel de valoración } \\
\hline & Alto & Medio & Bajo \\
\hline Planeación de actividades & $\begin{array}{l}\text { *El estudiante analiza } \\
\text { la situación problema } \\
\text { y plantea estrategias } \\
\text { para resolverlo. }\end{array}$ & $\begin{array}{l}\text { *El estudiante } \\
\text { entiende el } \\
\text { problema y aporta } \\
\text { algunas opciones } \\
\text { para llevarlo a cabo. }\end{array}$ & $\begin{array}{l}\text { *El alumno } \\
\text { lee el problema y } \\
\text { realiza } \\
\text { participaciones } \\
\text { espontaneas. }\end{array}$ \\
\hline & *El alumno colabora & *El alumno colabora & ${ }^{*}$ El alumno no \\
\hline & con sus compañeros, & espontáneamente y & colabora y no \\
\hline Deseo de Aprender & $\begin{array}{l}\text { fomenta el respeto y } \\
\text { se encuentra } \\
\text { motivado. }\end{array}$ & se muestra apático. & $\begin{array}{l}\text { muestra interés en } \\
\text { participar en las } \\
\text { actividades. }\end{array}$ \\
\hline Reflexión Crítica & $\begin{array}{l}\text { *El estudiante domina } \\
\text { el tema e investiga } \\
\text { para profundizar más. }\end{array}$ & $\begin{array}{l}\text { *El estudiante } \\
\text { muestra dominio } \\
\text { parcial del tema. }\end{array}$ & $\begin{array}{l}{ }^{*} \text { El estudiante } \\
\text { muestra un escaso } \\
\text { dominio del tema. }\end{array}$ \\
\hline
\end{tabular}

Tabla 6: Rúbrica de valoración cualitativa para las habilidades de autodirección durante la aplicación de la estrategia didáctica Aprendizaje Basado en Proyectos.

El instrumento "Observación en el aula" fue aplicado a cada uno de los alumnos individual y por equipos con el objetivo de recopilar mayor información sobre las tres dimensiones estructuradas en la rúbrica. La participación del docente fue moderada, por lo que existió un rol equilibrado entre las actividades dentro y fuera de la investigación; únicamente se le proporcionaba apoyo a los estudiantes cuando se requería o para brindar asesoría en algunos temas.

Las tres variables evaluadas en la rúbrica fueron planteadas de tal manera que abordara el proceso de autodirección del aprendizaje de los estudiantes durante el proceso de aplicación de la estrategia ABPr y las actividades que lo conforman. Las variables son: la Planeación de Actividades (PA), referida a las habilidades cognitivas expuestas; el Deseo de Aprender (DA), referida a toda la actividad afectiva; y la variable de Reflexión Crítica (RC), la cual implica una metacognición para la mejora de los aprendizajes.

Los resultados del instrumento cualitativo, se codifican de manera que se puedan extraer las actitudes, conocimientos y habilidades de los estudiantes, codificando así las respuestas en cada una de las dimensiones de la rúbrica específica. Valenzuela y Flores (2011), establecen que, en el análisis cualitativo, se considera al investigador como experto en el proceso de indagación desde una perspectiva naturalista, capaz de realizar una interpretación adecuada de los resultados obtenidos apegada a los objetivos del estudio.

Durante las actividades del proyecto se llevaron a cabo dos rúbricas del instrumento cualitativo; la primera consistió en los resultados individuales de cada alumno, mientras que la segunda se enfocó en el trabajo que realizaron los alumnos colaborativamente con su equipo de trabajo. Es importante recordar que el estudio se elaboró en un grupo de 18 alumnos. Para una mayor comprensión de los resultados, se especifica la información en la tabla 7. 


\begin{tabular}{cccc}
\hline \multirow{2}{*}{ Nivel de Desempeño } & Planeación de & Deseo de Aprender (DA) & Reflexión Crítica (RC) \\
& Actividades (PA) & 9 & 6 \\
Alto & 7 & 6 & 7 \\
Medio & 3 & 3 & 5 \\
Bajo & 8 & 9 & \\
\hline
\end{tabular}

Tabla 7: Número de alumnos por nivel de desempeño y dimensión del instrumento cualitativo.

En los resultados obtenidos, se puede percibir que las dimensiones aplicadas individualmente resaltaron las habilidades que los estudiantes utilizaron durante las actividades; en la dimensión PA se demostró que 7 alumnos utilizaron sus habilidades de búsqueda de información para la recolección de datos, analizaron ampliamente el problema y elaboraron estrategias que lo ayudaron a resolver todos los retos planteados. Los estudiantes reflexionaron acerca de las posibles soluciones buscando herramientas o fuentes que les permitieran encontrar respuestas a las preguntas planteadas por ellos mismos. Esta dimensión fomento en los alumnos una total reflexión acerca de "que querían lograr" " "que tenían que hacer para lograrlo".

En la dimensión DA hubo un gran número de alumnos en el nivel de desempeño alto, categorizándolo como la dimensión con mayor puntaje por los estudiantes durante el instrumento cualitativo. La mayor parte de los alumnos colaboraron con sus compañeros mostrándose totalmente empáticos y fomentando el respeto durante las actividades realizadas. Un aspecto relevante en esta dimensión fue el hecho de que los estudiantes eligieran a su pareja para trabajar en equipo; los estudiantes colaboraron mejor con los compañeros con los que tienen una mayor confianza para realizar las tareas descritas.

Finalmente, en la dimensión RC, se detectaron 7 alumnos en el nivel de desempeño medio, no habiendo tanta diferencia entre el nivel alto. Gracias a recolección de datos de un tema, los alumnos lograron dominar el tema e inclusive existieron casos en que los mismos estudiantes buscaban más información fuera del contexto escolar observando revistas o videos por Internet acerca de su tema de investigación.

La segunda rúbrica aplicada, consistió en rescatar los resultados de las tres dimensiones específicas organizados en equipos. Cabe aclarar que los equipos se estructuraron libremente, es decir, cada alumno eligió una pareja con la cuál trabajar, estableciéndose equipos de dos personas para conformar nueve equipos. Cada uno de los equipos fue observado de manera neutral durante la realización de las actividades; los resultados se muestran en la tabla 8.

\begin{tabular}{cccc}
\hline \multirow{2}{*}{ Nivel de Desempeño } & Planeación de & Deseo de Aprender (DA) & Reflexión Crítica (RC) \\
& Actividades (PA) & 4 & 5 \\
Alto & 3 & 3 & 4 \\
Medio & 5 & 2 & 0 \\
Bajo & 1 & 2 & \\
\hline
\end{tabular}

Tabla 8: Número de equipos por nivel de desempeño y dimensión del instrumento cualitativo.

Los resultados establecen que los equipos variaron en cada una de las dimensiones; sin embargo, se presentaron mejores resultados en comparación con la evaluación individual. Esto debido a la comunicación y trabajo en equipo 


\section{taphiya $\mathbf{4 6}$}

que cada pareja establecía y el compañerismo que utilizaban para realizar cada una de las actividades propuestas.

La dimensión PA, se muestra con 5 equipos en el nivel de desempeño medio demostrando que es relevante la postura y habilidades de cada alumno para portar ideas en la resolución de problemas de manera organizada y disciplinada (Cázares, 2009); el trabajo colaborativo en el aula es una de las habilidades más relevantes durante su proceso escolar, por lo que la aplicación del proyecto permitió que los alumnos expusieran sus ideas colaborativamente con las de otros en busca de un mismo resultado. En el nivel de desempeño bajo se coloco únicamente un equipo, el cual no mostraba iniciativa e interés por la resolución de problemas presentes al realizar las tareas establecidas.

La dimensión DA muestra que 4 equipos lograron obtener un nivel de desempeño alto, las actividades fueron realizadas con una actitud abierta hacia la producción de tareas y con un alto grado de compañerismo y respeto mutuo con sus compañeros. Los estudiantes demostraron un interés por obtener conocimientos sobre el tema que ellos eligieron definiendo como principal factor la voluntad individual para aprender a construir lo que les interesa (Cázares, 2009).

Por último, la dimensión RC mostró un alto grado de desempeño en 5 equipos y un desempeño medio en 4 equipos; lo cual demuestra que los equipos conformados demostraron un interés propio por seguir investigando sobre el mismo tema aumentando su nivel de conocimiento y practicando sus habilidades de búsqueda. De igual manera, los estudiantes demostraron dominar el tema gracias a la recopilación de datos hecha por ellos mismos.

Al evaluar la dimensión afectiva, cognitiva y metacognitiva, desde la perspectiva cualitativa y cuantitativa, se puede argumentar que los resultados promedios favorecen de forma estadísticamente significativa la mejora en la competencia de autodirección del aprendizaje. Los resultados obtenidos en la presente investigación, muestran a un grupo de estudiantes de educación primaria que han enfrentado el análisis de una situación de interés propia apropiadas al contexto escolar en el que se desenvuelven.

Los resultados recopilados en ambos tests demostraron que los estudiantes mostraron una actitud abierta por desarrollar un tema de su interés; durante las actividades se planteo que los alumnos realizaran una búsqueda de información de un tema de interés para ellos y para sus compañeros, plantearon preguntas y recolectaron información para realizar un escrito. Posteriormente realizaron apoyos gráficos para la exposición frente a sus compañeros.

Las dimensiones evaluadas en ambas pruebas (cuantitativa y cualitativa) demostraron que hay diferencia significativa en el proceso de autodirección del aprendizaje de los estudiantes durante el proceso de aplicación de la estrategia ABPr. Se puede argumentar que existió un amplio panorama en la triangulación de resultados obtenidos en la investigación; con el resultado de ambos tests se aumentó la validez del estudio y la consistencia de los hallazgos presentados.

\section{Discusión}

De acuerdo con el concepto de aprendizaje autodirigido utilizado por Cázares (2009), el interés es una parte importante durante el proceso de aprendizaje de los alumnos; sí la persona muestra interés en las actividades que realiza, logrará obtener el conocimiento necesario para la consecución de metas. En la presente investigación, los alumnos demostraron entusiasmo al elegir un tema de interés propio, lo que favoreció al desarrollo de sus habilidades para la obtención de resultados mostrando una mejora en su proceso de aprendizaje individual y colectivo. Asimismo, el uso de la estrategia ABPr permitió que los estudiantes generaran nuevas experiencias de organización de conocimientos a partir de un apoyo mutuo entre los equipos de trabajo tal como lo establece el autor Domínguez Chillón (2000).

\section{|34}


Con base a los resultados obtenidos en la evaluación cuantitativa y cualitativa del presente estudio, se pudo percibir una diferencia menor en el deseo de aprender y en el manejo de emociones, en las cuales se pretendía que los estudiantes lograrán efectuar un deseo por seguir aprendiendo de manera autónoma sobre los temas establecidos aumentando su motivación por el aprendizaje.

Estas acciones fueron ejecutadas sólo por algunos de los estudiantes, mientras que el resto del grupo quería pasar al siguiente tema sin detenerse a buscar más información relevante del tema. Olivares y López (2015), argumentan que el desarrollo de la capacidad autodirigida implica una motivación y un deseo de aprender constante para la construcción de conocimientos y habilidades que conlleva a la resolución de problemas para el logro de los objetivos de aprendizaje; mientras que Palmer, Montaño y Palou (2009) contemplan que debe existir un balance entre los conocimientos teóricos y prácticas, así como las actitudes que van del saber y saber hacer al saber ser o estar.

Bajo esta perspectiva, el presente estudio aporta cambios en los procesos de aprendizaje en un aula de segundo año de educación primaria, en el cuál se abordaron temas de interés de los alumnos para la creación de un proyecto. Cabe destacar la importancia de que los estudiantes generaron experiencias que les permitieron obtener conocimientos a raíz de su propio trabajo; esta investigación tuvo como propósito ampliar su rango permitiendo que los docentes implementen nuevas estrategias de enseñanza permitiendo que sus estudiantes se involucren en una educación totalmente centrada en la construcción de competencias produciendo una nueva generación de conocimientos en las instituciones formando alumnos autodirigidos y eficaces en los procesos de enseñanza.

En el proceso de autodirección, el papel del docente debe establecer como objetivo central en la educación el desarrollo de las actitudes y habilidades que apoyan el aprendizaje permanente autodirigido en los estudiantes (Guglielmino, 2008).

Los maestros de cada grupo, deben asumir la importancia de proporcionar al niño situaciones retadoras dentro de los parámetros curriculares establecidos, brindando una motivación en el aula y despertando la curiosidad por el querer aprender. Para conocer las ideas de los niños es importante explorar sus acciones y sus respuestas a preguntas cotidianas, de esta manera el profesor puede alentarlos a crear ideas explícitas de un tema (Glauert, 1998); se trata de convertir una lección en una experiencia divertida e interesante donde el alumno ponga en juego sus conocimientos y habilidades generando competencias para la vida.

El docente es el facilitador de escenarios adecuados y recursos pertinentes para la construcción de aprendizaje, su papel en la educación es ser una guía que facilite a los estudiantes situaciones que les permita construir conocimientos propios. Marco Silva (2005) menciona que "el profesor no transmite el conocimiento, sino que facilita el acceso a dominios de conocimientos de modo expresivamente complejo y, al mismo tiempo, un entorno que garantiza la libertad y la pluralidad de las expresiones individuales y colectivas" (pág. 236). 


\section{Bibliografía}

Alvira Alvira, T. (1992): El Ramiro de Maeztu, Pedagogía Viva. Madrid: Ediciones Rialp. 293 págs.

CLARDY, A. (2000). Learning on Their Own: Vocationally Oriented Self-Directed Learning Projects. Human Resource Development Quarterly, 11(2), 105-125.

CÁzARES, Y. M. (2009). La autodirección, la persona autodirigida y sus componentes: Definiciones conceptuales. El Tintero, 9(38), 1-4.

ARIAS Molina, I.E. (2015). El Aprendizaje Basado en Proyectos, y su relación con el Pensamiento Crítico en estudiantes de sexto grado de primaria. Puebla, México: Universidad Tecvirtual, Tecnológico de Monterrey. (Tesis inédita de maestría).

Domínguez ChilLón, G. (2000). Qué entendemos por proyectos de trabajo, en la educación preescolar. En G. Domínguez Chillón. Proyectos de trabajo. Una escuela diferente. (pp. 27-40). Barcelona: La Muralla.

EquiPO dE EDUCACIÓN INFANTIL y PRIMER CICLO DE PRIMARIA del CP ANTZUOLA. (2001, mayo-junio). Niños y niñas investigadoras: ¿de qué hablamos? Aula Infantil, 1(1), 7-16.

FeBreR, M.D. (2001, mayo-junio). La Tierra en el Universo: ¿es eso un proyecto? Aula de Infantil, 1(1), 27-33.

GLAUERT, E. (1998). La ciencia en los primeros años. En I. Siraj-Blatchford. A curriculum development handbook for early childhood educators (pp. 77-91). Londres: Trentham Books Limited.

GuAYACUNDO RamoS, A.P. (2015). El aprendizaje basado en proyectos para favorecer el pensamiento crítico en alumnos de primaria publica en la ciudad de Bogotá, Colombia. Bogotá, Colombia: Universidad Tecvirtual, Tecnológico de Monterrey. (Tesis inédita de maestría).

GugliELmino, L.M. (2008). Why Self-directed Learning? International Journal of Self-Directed Learning, 5(1), 1-14.

HeRnÁndeZ, R.; FeRnández, C. y BaPtista, P. (2010). Metodología de la Investigación. México: McGraw Hill.

MartíneZ, M.R. y McGrath, D. (2013). How can schools develop self-directed learners? The Phi Delta Kappan, 95(2), 2327.

OLIVARES, S.L. y LópEZ, M.V. (2015). Medición de la autopercepción de la autodirección en estudiantes de medicina de pregrado. Investigación en Educación Médica, 4(14), 75-80.

Olivares OlivareS, S. (2015). Business Graduate Skills; Competency-Based Model. En M. Khan. Diverse Contemporary Issues Facing Business Management Education (pp. 25-51). Hershey, PA: IGI Global.

OKudA Benavides, M. y Gómez-Restrepo, C. (2005). Métodos en investigación cualitativa: triangulación. Revista Colombiana de Psiquiatría, XXXIV(1), 118-124.

Palmer, A.; Montaño, J.J. y Palou, M. (2009). Las competencias genéricas en la educación superior. Estudio comparativo entre la opinión de empleadores y académicos. Psicothema, 21(3), 433-438.

ParRA, J.; Cerda, C.; LÓPez-VARGAS, 0. y SAIZ, J.L. (2014). Género, autodirección del aprendizaje y desempeño académico en estudiantes de pedagogía. Educación y Educadores, 7(1), 91-107.

SALGADO, F.; CORRALES, J.; MuÑoz, L. y DelGAdo, J. (2012). Diseño de programas de asignaturas basados en competencias y su aplicación en la Universidad del Bío Bío, Chile. Revista chilena de ingeniería, 20(2), 267-278. 


\section{tapbiya $\mathbf{4 6}$}

SEP. (2011c). Programas de estudio 2011. Guía para el Maestro. Educación Básica. Primaria. Segundo grado. México, D.F.

SILvA, M. (2005). Educación Interactiva: Enseñanza y aprendizaje presencial y online. Barcelona: Gedisa, S.A.

TOBón, S. (2006). Aspectos básicos de la formación basada en competencias.

http://maristas.org.mx/gestion/web/doctos/aspectos_basicos_formacion_competencias.pdf

TonnuCl, F. (1996). El niño y la ciencia. En G.K. (trad.). Con ojos de maestro (pp. 85-107). Buenos Aires: Troquel.

Tonnucl, F. (2001). ¿Cómo introducir la investigación escolar? En F. Tonnuci. Investigación en la Escuela (pp. 39-50).

ValenzuelA, J.R. y FLoRES, M. (2011). Fundamentos de investigación educativa, Vol. 2: El proceso de investigación educativa. Monterrey, Nuevo León, México.

VILLA, A. y POBLETE, M. (2007). Aprendizaje basado en competencias: Una propuesta para la evaluación de competencias genéricas. Bilbao, España: Ediciones Mensajero S.A.U.

Vives-Varela, T.; Durán-CárdenAS, C.; Varela-Ruíz, M. y Fortoul van der Goes, T. (2013). La autorregulación en el aprendizaje, la luz de un faro en el mar. Investigación en Educación Médica, 3(9), 34-39. 


\section{taphiya $\mathbf{4 6}$}

\section{Resumen.}

La educación por competencias permite a los estudiantes enfrentar y resolver situaciones problemáticas que les plantea el entorno que los rodea. La presente investigación tuvo como objetivo determinar la forma en que la estrategia didáctica Aprendizaje Basado en Proyectos (ABPr) permite desarrollar la competencia de aprendizaje autodirigido en alumnos de segundo grado de educación primaria. La población elegida para la elaboración de esta investigación se enfoca en 18 alumnos de segundo grado de educación primaria inscritos en un colegio particular ubicado en la localidad de San Luis Potosí, México. La estrategia ABPr se planteó durante una serie de actividades donde los estudiantes realizaron la búsqueda de información de un tema de interés. Los instrumentos utilizados fueron, como instrumento cuantitativo, la sección de Autodirección del Cuestionario de Competencias Genéricas Individuales (CCGI) de Olivares y López (2015); y como instrumento cualitativo la Observación en el aula. Los resultados fueron evaluados con la estadística t student, la cuál indicó un p valor de 0.04, comprobando que hay una diferencia significativa en el proceso de autodirección favoreciendo el aprendizaje durante el proceso de aplicación de la estrategia ABPr.

Palabras clave. Aprendizaje Autodirigido, Educación Básica, Proyectos Educativos, Competencias.

\section{Abstract.}

Education by competences allows students to face and solve problematic situations related to real contexts. The purpose of this research was to determine how the Project-Oriented learning strategy (POL) may facilitate the development of self-direction competence in second grade students from elementary education. The population where 18 second grade students from a private school located in San Luis Potosí, Mexico. The POL strategy was applied during a series of activities where the students searched information on a topic of interest. The selected instruments were, as a quantitative instrument, the Self-Direction section of the Generic Skills Test by Olivares and López (2015); and as a qualitative instrument, observation in the classroom. The results were analyzed with the t student statistic, which indicated a p value of 0.04 , which reflected a significant difference of self-direction during the application process of the POL strategy.

Key-words. Self-direction competence, Elementary education, Project-education, Competences.

Abril Paulina López Colón

Secretaria de Educación del Gobierno del Estado

Licenciada en Educación Preescolar

Docente frente a grupo en Villa de Reyes, SLP

aprilpau_94@hotmail.com

Silvia Lizeth Olivares Olivares

Decana Asociada Académica Escuela de Medicina y Ciencias de la Salud

Tecnológico de Monterrey

solivares@itesm.mx 


\section{tapbiya $\mathbf{4 6}$}

Miriam Lizzeth Turrubiartes Corolla

Coordinadora de Innovación Académica Escuela de Medicina y Ciencias de la Salud

Tecnológico de Monterrey

mturrubiates@itesm.mx 